\title{
Distributed Opportunistic Spectrum Access with Unknown Population
}

\author{
Marjan Zandi and Min Dong \\ Department of Electrical and Computer Engineering \\ University of Ontario Institute of Technology, Ontario, Canada \\ Email: \{marjan.zandi, min.dong\}@uoit.ca
}

\begin{abstract}
We consider a cognitive radio network where $M$ secondary users compete with each other to access one of the $N$ available channels. Channel availability statistics are assumed to evolve as i.i.d. Bernoulli random processes with means unknown to the secondary users. In addition, the number of secondary users $M$ is unknown to each user. The main objective here is to design a distributed online learning and access policy which maximizes the total throughput of the secondary users. It has previously been shown that this problem can elegantly be modeled as a decentralized multi-armed bandit (DMAB) problem when $M$ is known. We propose a truly decentralized online learning algorithm based on DMAB problem for unknown $M$. We show that using distributed access policies with wrong knowledge of $M$ results in linear growth of regret, and underestimation incurs more significant loss than overestimation does. For distributed online learning of $M$, we propose a dynamic thresholding method, where the thresholds are dynamically determined using virtual systems built upon the current estimates of mean channel availabilities. Our algorithm allows both overestimation and underestimation in estimating $M$ over time, and thus is capable of tracking the population change of secondary users.
\end{abstract}

\section{INTRODUCTION}

One of the main challenges in cognitive radio networks is to design dynamic spectrum access to efficiently utilize the spectrum. A hierarchical cognitive radio network consists of primary users who are licensed to use the spectrum and the secondary users who opportunistically use the idle channels that are not occupied by the primary users. The channel availability statistics of the primary network are typically unknown to the secondary users. Through limited spectrum sensing, the secondary users search for idle channels and make decisions based on observation histories for channel access. In many scenarios, the secondary users are uncoordinated, ad hoc, and/or dissimilar. Thus, designing distributed policy for spectrum access among secondary users, where no information exchange or access arrangement among users, to maximize the total throughput of secondary users is critical. In this case, the challenges involved in dynamic spectrum access not only include online learning of the primary channel statistics based on local sensing observations, but also the distributed mechanism to resolve collisions among secondary users.

Consider a cognitive radio network with $N$ independent channels and $M$ secondary users, where $N \geq M$. For centralized scheduling of users' access, the problem of selecting the best $M$ channels to maximize the throughput of secondary users under unknown channel availability statistics can be formulated as the classical Multi-Armed Bandit (MAB) problem [1]-[3]. In this case, the problem is to design a policy to sequentially choose $M$ plays of $N$ arms with i.i.d. rewards over time. The performance of a MAB policy is evaluated by a metric called regret, defined as the difference in total expected rewards by the optimal choice and that by a given policy. For distributed access by the secondary users, the problem formulation can be viewed as the decentralized MAB problem. In contrast to the classic MAB problem, for decentralized MAB problem, $M$ players compete over $N$ arms. When multiple secondary users pick the same arm, collision occurs resulting in lost rewards. To address this problem, particularly arisen in dynamic spectrum access, a few decentralized learning and access policies were developed recently [4]-[6]. These policies use different mechanisms to achieve "coordination" among secondary users to orthogonalize their access to the $M$-best channels, and all achieve logarithmic growth of regret. Common to all these proposed distributed algorithms, the number of secondary users, $M$ is assumed known to each secondary user. This information is utilized in determining the access decision to one of the $M$-best channels. Thus, although these algorithms are distributed in terms of learning of channel availability statistics based on local observation histories, secondary users share the common knowledge of the user population. In a practical dynamic environment, such knowledge may not be known and needs to be estimated and tracked at each secondary user in order to implement the distributed access policy.

In this paper, we consider such a truly distributed spectrum access environment where both population of secondary users and channel availability statistics are unknown to secondary users, and population may change over time. We aim at developing a distributed mechanism for joint distributed access and user population estimation and tracking. Our particularly focus is on extending $\rho^{\mathrm{RAND}}$ policy proposed in [5] to the scenario with unknown user population and its online estimation. We first show that using distributed access policies with wrong knowledge of $M$ will result in linear growth of regret over time. In particular, the loss due to underestimation is more significant than that of overestimation, reflected in the rate of growth in regret. For distributed online learning of $M$, we propose a dynamic thresholding method in which collision counts are tested against thresholds in estimating $M$. The thresholds are dynamically adjusted using virtual systems built upon the current estimates of mean channel availabilities. Our algorithm allows both overestimation and underestimation of 
$M$ over time, and thus is capable of tracking the population change in a dynamic network environment.

Extensive research has been conducted in MAB problems. Both classical results for single play [1] and multi-play [2], [3] provide policies that are efficient in regret. Simple indexbased policies were proposed [7], [8] which have logarithmic growth of regret. Motivated by dynamic spectrum access, decentralized policies among multiple players are proposed [4] [6], all achieving logarithmic growth of regret. In [4], a TDFS policy is proposed to orthogonalize secondary users in a TDM fashion, which requires certain coordination among secondary users with the knowledge of $M$. In [5], a simple $\rho^{\text {RAND }}$ policy is proposed to randomize each user's pick of the $M$-best channels. In [6], a DLF policy is proposed which is shown to have an order-optimal scaling with respect to $M$ and $N$. However, the distributed collision-resolving mechanism relies on the knowledge of $M$ and each secondary user's assigned id. Consequently, the DLF policy cannot be implemented in an unknown population environment. Closely related to our work, [5] also considered the scenario when $M$ is unknown, and proposed $\rho^{\mathrm{EST}}$ policy based on $\rho^{\mathrm{RAND}}$ policy. The $\rho^{\mathrm{EST}}$ policy uses the idea of testing collusion counts by a secondary user against thresholds to provide an estimate of $M$, but only at the conceptual level without any specific method given. A more detailed account of the differences between our work and [5] is given in Section IV-B.

\section{NETWORK MOdEL}

We consider a cognitive radio network, where the spectrum consists of $N$ orthogonal channels that are licensed to a slotted primary network. We consider a secondary network with $M$ (secondary) users independently searching for the idle channels among the $N$ channels, where $M \leq N$. Let $X_{i}(n)$ be the availability state of the $i$ th channel in the primary network at slot $n$, with

$$
X_{i}(n)=\left\{\begin{array}{l}
1, \text { channel } i \text { is available in slot } n \\
0, \text { o.w. }
\end{array}\right.
$$

We assume the availability statistic for each channel $i$ in the primary network evolves as an i.i.d. Bernoulli random process over time, with the mean $\theta_{i} \in[0,1]$. That is, for all $n, X_{i}(n) \sim \operatorname{Bernoulli}\left(\theta_{i}\right)$, where $\theta_{i}=E\left[X_{i}(n)\right]$, for $i=1, \cdots, N$. We assume $\theta_{i}$ 's are distinct to each other and are unknown to the secondary users. We denote the mean vector as $\boldsymbol{\theta} \triangleq\left[\theta_{1}, \theta_{2}, \ldots, \theta_{N}\right]^{T}$. In addition, we assume the total number of secondary users $M$ is also unknown to all secondary users. At the beginning of each slot $n$, each secondary user selects a channel to sense, and if available, the user will access the channel. Here, perfect channel sensing is assumed at all secondary users. Note that, the secondary users can use the sensing outcome and history to learn the availability statistics over time. Thus, although $\boldsymbol{\theta}$ is unknown to the secondary users initially, it will be learned over time by each secondary user.

We assume a collision model for multiple access by the secondary users. If more than one secondary user selects the same channel $i$ to transmit, then no one gets through, and each colliding user gets zero reward. Otherwise, the sole secondary user accessing channel $i$ will get reward 1 .

System Regret: Under any access policy, a measure called regret is used as a common performance metric for design and comparison. It defined as the difference between the total expected number of successful transmissions (throughput) under an ideal scenario, where $\boldsymbol{\theta}$ is known and the $M$-best channels are always orthogonally allocated to the secondary users, and the expected number of successful transmission obtained by a given policy, i.e.,

$$
R(n, \boldsymbol{\theta}, M) \triangleq n \sum_{k=1}^{M} \theta_{k^{*}}-\sum_{i=1}^{N} \sum_{j=1}^{M} \theta_{i} E\left[S_{i}^{j}(n)\right]
$$

where $k^{*}$ represents index of the $k$ th highest element of the $\boldsymbol{\theta}$, and $S_{i}^{j}(n)$ denotes the number of times, up to current slot $n$, that secondary user $j$ is the only user to sense channel $i$.

Given the above model, a main problem is how to design decentralized policy that minimizes the regret, with no exchange of information among them. For a truly distributed access policy, each secondary user will select a channel to access based on its own estimate of $\hat{M}$ and the mean availability of channels $\hat{\theta}_{i}^{j}\left(T_{i}^{j}(n)\right)$.

\section{Decentralized Spectrum Access Policies}

The existing decentralized policies [4]-[6] can be viewed as variant distributed extensions of the UCB1 algorithm proposed in [7], which is a sample-mean based index policy for the single user case. In the UCB1 algorithm, an index is assigned to every channel. The assigned index is a statistic which is based on the estimated sample mean of channel $i$ and the total number of times that channel $i$ has been visited up to the current time slot $n$. Let $T_{i}^{j}(n)$ denote the number of times that the secondary user $j$ senses channel $i$ up to time slot $n$. If the secondary user $j$ picks channel $i$ to sense at time slot $n$, then it obtains the value of $X_{i}(n)$ and records this value as $X_{i}^{j}\left(T_{i}^{j}(n)\right)$. Let $\mathbf{X}_{i}^{j}(n) \triangleq\left[X_{i}^{j}(1), \cdots, X_{i}^{j}\left(T_{i}^{j}(n)\right)\right]^{T}$ be the vector holding the sensing observations of secondary user $j$ for channel $i$ up to time slot $n$. With these sensing observations, secondary user $j$ can estimate $\theta_{i}$, the mean availability of channel $i$, at time $n$ as

$$
\hat{\theta}_{i}^{j}\left(T_{i}^{j}(n)\right) \triangleq \frac{1}{T_{i}^{j}(n)} \sum_{k=1}^{T_{i}^{j}(n)} X_{i}^{j}(k) .
$$

Each secondary user $j$ obtains an index called g-statistic for all the channels, $i=1, \cdots, N$, as

$$
I_{j}(i, n) \triangleq \hat{\theta}_{i}^{j}\left(T_{i}^{j}(n)\right)+\sqrt{\frac{2 \log n}{T_{i}^{j}(n)}}
$$

for $j=1, \cdots, M$. In the single user case $(M=1)$, the above index will be used to rank all the channels and the user then selects the channel with the highest index at time $n$. When there are multiple secondary users, each user computes its own index vector of the channels $\mathbf{I}_{j}(n) \triangleq\left[I_{j}(1, n), \cdots, I_{j}(N, n)\right]^{T}$ 
based on its own observation history. Then, with certain distributed coordination designed differently by [4]-[6], each user will select a $k$ th-best channel (i.e., the channel with the $k$ th highest ranking in $\left.\mathbf{I}_{j}(n)\right)$ to access, to ensure that the secondary users choose different channels but within the first $M$-best channels. Our proposed policy is closely related to $\rho^{\text {RAND }}$ policy proposed in [5], which is explained below:

1) Select channel to sense and access: At every time slot $n$, each secondary user $j$ obtain its ranking vector of primary channels $\mathbf{I}_{j}(n)$. It then selects the $r_{j}$ thbest channel among the $M$-best channels to sense, where $r_{j}$ is drawn from a uniform distribution: $r_{j} \stackrel{i . i . d \text {. }}{\sim}$ Uniform $(M)$, for $j=1 \cdots, M$. Let $\sigma\left(r_{j}, \mathbf{I}_{j}(n)\right)$ be the channel index of the $r$ th highest rank in $\mathbf{I}_{j}(n)$. If the channel is available $X_{\sigma\left(r_{j}, \mathbf{I}_{j}(n)\right)}=1$, then the user access the channel.

2) Reselect channel under collision: Each user $j$ uses an acknowledgement for collision feedback. Let $\zeta_{j}(i, n) \in$ $\{0,1\}$ denote such acknowledgment for user $j$ on channel $i$, with $\zeta_{j}(i, n)=1$ being collision among secondary users. Each user will redraw its rank $r_{j} \sim \operatorname{Uniform}(M)$ only if there is collision in the previous transmission, otherwise, it will keep using the rank $r_{j}$ generated previously.

\section{DECENTRALIZED SPECTRUM ACCESS WITH UNKNOWN SU POPULATION}

When $M$ is unknown, each secondary user $j$ needs to obtain an estimate $\hat{M}_{j}$. It will then choose one of the $\hat{M}_{j}$ best channels to access. In the following, we will propose a dynamic thresholding policy $\pi_{\mathrm{DT}}$, incorporating dynamic estimation of $M$ and the distributed access in $\rho^{\text {RAND }}$ under $\hat{M}_{j}$.

\section{A. Linear Growth of Regret for $\hat{M}_{j} \neq M$}

We first show that a fixed but inaccurate estimate $\hat{M}_{j} \neq M$ will lead to the linear growth of regret. This is in contrast to the logarithm growth under the perfect knowledge of $M$.

- Underestimation: Through online learning processes, the secondary users gather more information and obtain better estimate $\hat{\boldsymbol{\theta}}$ of $\boldsymbol{\theta}$. It can be shown that, the probability of events that all users have the $M$-best channels asymptotically goes to 1 . Among $M$-best channels, the users will try to orthogonalize the channel to access with each other. If only one user underestimate the user population, i.e., $\hat{M}_{j}<M$, for some $j$, there will be at least one collision among users every slot, provided all users share the same $M$-best channels. Thus, a smaller value of $\hat{M}_{j}$ will cause a linear growth of regret $O(n)$. The actual regret is lower bounded by this best scenario.

- Overestimation: Again, the probability of events that all users have the $M$-best channels asymptotically goes to 1. Overestimating $M$ leads to the possibility of choosing the next $(\hat{M}-M)$-best channels, instead of $M$-best channels. This results in the difference in mean channel availabilities as compared to the optimal choice, which translates into linear growth of regret $O(n)$.
Even though both underestimation and overestimation lead to linear growth of regret, comparing the two, overestimation leads to lower regret than underestimation (i.e., smaller leading coefficient of growth rate). To see this, for $\hat{M}_{j}<M$ case, collisions lead to zero reward (throughput); on the other hand, choosing from non $M$-best channels leads to an expected non-zero reward with the loss bounded by the difference of the mean availability of the $\hat{M}$ th-best channel and the best channels. We will demonstrate in simulation results the difference in performance loss in terms of regret between overestimation and underestimation cases.

\section{B. Population Estimation through Thresholding}

In a practical system, we need to dynamically estimate the user population, based on the observation history, to improve our knowledge of $M$. In [5], a $\rho^{\mathrm{EST}}$ algorithm is proposed under unknown $M$ which is a modified policy of $\rho^{\text {RAND }}$ policy. In $\rho^{\mathrm{EST}}$ algorithm, with the total transmission horizon $T$ known, the estimate $\hat{M}_{j}$ for user $j$ is updated by comparing the number of collisions so far to a threshold $\psi\left(T, \hat{M}_{j}\right)$. The idea is that if $\psi\left(T, \hat{M}_{j}\right)$ is chosen properly, then for $\hat{M}_{j}<M$, the collision build-up will make the collision count exceed $\psi\left(T, \hat{M}_{j}\right)$, serving as a mechanism to increase $\hat{M}$. However, there are three aspects in $\rho^{\mathrm{EST}}$ that were not discussed:

- The policy and its property critically depends on the threshold $\psi\left(T, \hat{M}_{j}\right)$ setting. However, no explicit method for setting the thresholds was discussed; instead, $\psi\left(T, \hat{M}_{j}\right)$ was assumed given.

- The policy only allows upward increase of $\hat{M}_{j}$. Thus, to ensure accurate estimation, the threshold needs to be set large to avoid overestimation. This leads to slow learning. For finite horizon, since the user population will mostly be underestimated, it results in large regret as we have shown in the previous section.

- The policy cannot adapt to changing environment when $M$ varies. This is because the one-way upward increase mechanism of $\hat{M}_{j}$.

In this paper, we try to address the above three issues in our policy design by proposing a method to set threshold, and dynamic updating $\hat{M}_{j}$ which allow users to track $M$ in a changing environment.

Let $n_{o}$ be the time slot of previous $\hat{M}_{j}$ update. Given the collision indicator $\zeta_{j}(i, t)$ at slot $t$ on channel $i$, the total number of collisions experienced by secondary user $j$ up to the current slot $n$ can be obtained as

$$
K_{j}\left(n ; \hat{M}_{j}, \boldsymbol{\theta}\right)=\sum_{t=n_{o}+1}^{n} \sum_{k=1}^{\hat{M}_{j}} \zeta_{j}\left(\sigma\left(k, \mathbf{I}_{j}(t)\right), t\right) .
$$

Note that, besides being a function of $\hat{M}_{j}$, the total number of collisions $K_{j}\left(n ; \hat{M}_{j}, \boldsymbol{\theta}\right)$ is also a function of $\boldsymbol{\theta}$. Indeed, the distribution of entries in $\boldsymbol{\theta}$ will affect the accuracy in (4) that ranks the channel availabilities based on their estimates $\hat{\theta}_{i}^{j}\left(T_{i}^{j}(n)\right), i=1, \cdots, N$.

We will use a thresholding method, where the threshold reflects the expected collision counts when the the secondary 
user population is indeed the estimate $\hat{M}_{j}$. The collision counts $K_{j}\left(n ; \hat{M}_{j}, \boldsymbol{\theta}\right)$ will be tested against such threshold to determine whether to increase or decrease the current estimate $\hat{M}_{j}$.

\section{Dynamic Thresholding Policy $\pi_{D T}$}

As mentioned, the threshold for each secondary user $j$ should reflect the expected number of collisions under $\hat{M}_{j}$, i.e., $\mathrm{E}\left[K_{j}\left(n ; \hat{M}_{j}, \boldsymbol{\theta}\right)\right]$. To determine $\mathrm{E}\left[K_{j}\left(n ; \hat{M}_{j}, \boldsymbol{\theta}\right)\right]$, we need the mean availability vector $\boldsymbol{\theta}$ which is unknown. To address this issue, we propose a dynamic thresholding method using virtual systems. In this case, instead of being fixed, the threshold is dynamic based on current sample mean channel availability $\hat{\boldsymbol{\theta}}_{j}$, and thus it is a function of $n$. We denote this dynamic threshold at slot $n$ for user $j$ as $\Delta_{j}\left(\hat{M}_{j}, n\right)$.

1) Virtual System: For each secondary user $j$ at slot $n$, we consider a set of virtual systems: $\left\{\mathcal{S}_{j 1}(n), \ldots, \mathcal{S}_{j N}(n)\right\}$. Each virtual system $\mathcal{S}_{j u}(n)$ consists of $N$ primary channels and $u$ secondary users. Channel availability statistics for the $N$ primary channels is denoted as $\boldsymbol{\theta}_{j}^{\prime}=\left[\theta_{j 1}^{\prime}, \cdots, \theta_{j N}^{\prime}\right]^{T}$, which is set to be equivalent to $\boldsymbol{\theta}_{j}^{\prime} \equiv \hat{\boldsymbol{\theta}}_{j}(n)$, where

$$
\hat{\boldsymbol{\theta}}_{j}(n) \triangleq\left[\hat{\theta}_{1}^{j}\left(T_{1}^{j}(n)\right), \cdots, \hat{\theta}_{N}^{j}\left(T_{N}^{j}(n)\right)\right]^{T} .
$$

In other words, the virtual system is build upon the primary network using the current estimate of channel availability in the original true system, and installs $u$ secondary users in the system. For each virtual system $\mathcal{S}_{j u}(n)$, the secondary user population $u$ is known to every user $j^{\prime}$, and each user uses the $\rho^{\text {RAND }}$ policy for access, for $j^{\prime}=1, \cdots, u$. Similar to (5), the collision count from the beginning until the current slot $n^{\prime}$ is then given by

$$
K_{j^{\prime}}\left(n^{\prime} ; u, \boldsymbol{\theta}_{j}^{\prime}\right)=\sum_{t=1}^{n^{\prime}} \sum_{k=1}^{u} \zeta_{j^{\prime}}\left(\sigma\left(k, \mathbf{I}_{j^{\prime}}(t)\right), t\right)
$$

for $j^{\prime}=1, \cdots, u$.

2) Dynamic Thresholding $\Delta_{j}\left(\hat{M}_{j}, n\right)$ : With the virtual system that is created using the current estimate $\hat{\boldsymbol{\theta}}_{j}(n)$ for each secondary user $j$, we can dynamically obtain the threshold $\Delta_{j}\left(\hat{M}_{j}, n\right)$. Running $\rho^{\text {RAND }}$ policy on the virtual systems generated by secondary user $j$, the secondary users in the virtual system try to estimate their unknown $\boldsymbol{\theta}^{\prime}{ }_{j}$. After a given time horizon $T^{\prime}$, the average collision counts for the virtual systems can be obtained, which will be used to determine the threshold.

Denote $\bar{K}\left(n^{\prime}, u, \boldsymbol{\theta}_{j}^{\prime}\right)$ as the average collision counts for the virtual system $\mathcal{S}_{j u}(n)$, averaged over all users, i.e.,

$$
\bar{K}\left(n^{\prime} ; u, \boldsymbol{\theta}_{j}^{\prime}\right)=\frac{\sum_{j^{\prime}=1}^{u} K_{j^{\prime}}\left(n^{\prime} ; u, \boldsymbol{\theta}_{j}^{\prime}\right)}{u} .
$$

It serves as an estimate of the expected number of collision in the system with $u$ secondary users under $\rho^{\mathrm{RAND}}$ policy, i.e., $\mathrm{E}\left[K_{j^{\prime}}\left(n^{\prime}, u, \boldsymbol{\theta}_{j}^{\prime}\right)\right]$. It has been shown in [5] that the expected number of collisions under $\rho^{\mathrm{RAND}}$ has logarithmic growth, i.e., $\mathrm{E}\left[K_{j^{\prime}}\left(n^{\prime}, u, \boldsymbol{\theta}_{j}^{\prime}\right)\right]=O\left(\log n^{\prime}\right)$. Thus, we will set the dynamic threshold $\Delta_{j}(u, n)$ at slot $n$ in the true system using this estimate as

$$
\Delta_{j}(u, n)=\frac{\bar{K}\left(T^{\prime} ; u, \boldsymbol{\theta}_{j}^{\prime}\right)}{\log T^{\prime}}
$$

where $T^{\prime}$ is the time horizon used to run the virtual system.

To apply the virtual systems, at slot $n$, we following the following steps

1) In the original true system, a secondary user $j$ obtains its estimate $\hat{M}_{j}$;

2) User $j$ will then activate two virtual systems, $\mathcal{S}_{j \hat{M}_{j}}(n)$ and $\mathcal{S}_{j\left(\hat{M}_{j}-1\right)}(n)$, and compute $\Delta_{j}\left(\hat{M}_{j}, n\right)$ and $\Delta_{j}\left(\hat{M}_{j}-1, n\right)$ based on (8) and (9);

3) User $j$ will use $\Delta_{j}\left(\hat{M}_{j}, n\right)$ and $\Delta_{j}\left(\hat{M}_{j}-1, n\right)$ to test against $K_{j}\left(n ; \hat{M}_{j}, \boldsymbol{\theta}\right) / \log n$ to determine whether to increase or decrease $\hat{M}_{j}$ by 1 :

If $K_{j}\left(n ; \hat{M}_{j}, \boldsymbol{\theta}\right) / \log \left(n-n_{o}\right)>\Delta_{j}\left(\hat{M}_{j}, n\right), \hat{M}_{j}=$ $\hat{M}_{j}+1$;

Else if $K_{j}\left(n ; \hat{M}_{j}, \boldsymbol{\theta}\right) / \log \left(n-n_{o}\right)<\Delta_{j}\left(\hat{M}_{j}-1, n\right)$, $\hat{M}_{j}=\hat{M}_{j}-1$;

Else no change.

If at slot $n$, secondary user $j$ updates its estimate $\hat{M}_{j}$, the total collision count so far is discarded and secondary user $j$ starts new collision counts afterward, i.e., $n_{o}=n$ in (5). The details of the dynamic thresholding for population estimation and access policy is summarized in Algorithm 1.

From the above, we see that as $\hat{\boldsymbol{\theta}}_{j}(n) \stackrel{n}{\rightarrow} \boldsymbol{\theta}$, we have

$$
\frac{\mathrm{E}\left[K_{j^{\prime}}\left(T^{\prime} ; \hat{M}_{j}, \boldsymbol{\theta}_{j}^{\prime}\right)\right]}{\log T^{\prime}} \rightarrow \frac{\mathrm{E}\left[K_{j}\left(T^{\prime}-n_{0} ; \hat{M}_{j}, \boldsymbol{\theta}\right]\right)}{\log \left(T^{\prime}-n_{0}\right)},
$$

where the LHS expression is from the virtual system and the RHS expression is under the true system. Thus, the threshold in (9), for $u=\hat{M}_{j}$, provides an estimate of $\mathrm{E}\left[K_{j}\left(T^{\prime}-n_{0} ; \hat{M}_{j}, \boldsymbol{\theta}\right]\right) / \log \left(T^{\prime}-n_{0}\right)$ in the true system.

3) Implementation Aspects: From above, we see that we essentially obtain the threshold $\Delta_{j}\left(\hat{M}_{j}, n\right)$ based on the threshold generated from the proposed virtual systems. In the following, we discuss two parameters to be set in the actual system:

a) Time horizon $T^{\prime}$ : The time horizon $T^{\prime}$ in virtual system can be either set with a predetermined fixed value or it can be changed dynamically. For setting $T^{\prime}$ as a fixed value, it needs to be large enough to guarantee that $\mathrm{E}\left[K_{j^{\prime}}\left(T^{\prime} ; \hat{M}_{j}, \boldsymbol{\theta}_{j}^{\prime}\right)\right] / \log T^{\prime}$ has reached its steady value. This may require a large value of $T^{\prime}$, which will make the online population estimation process slow. On the other hand, we can dynamically determine $T^{\prime}$ : Based on the variation of $\bar{K}\left(T^{\prime} ; \hat{M}_{j}, \boldsymbol{\theta}_{j}^{\prime}\right) / \log T^{\prime}$ in (9) over $T^{\prime}$, we can determine whether the current $T^{\prime}$ is sufficient for determine the current threshold $\Delta_{j}\left(\hat{M}_{j}, n\right)$.

b) Threshold $\Delta_{j}\left(\hat{M}_{j}, n\right)$ updating frequency: In $\pi_{\mathrm{DT}}$ policy, every secondary user $j$ needs to activate two virtual systems to obtain the current value of $\Delta_{j}\left(\hat{M}_{j}, n\right)$ and $\Delta_{j}\left(\hat{M}_{j}-1, n\right)$, which can be slow and also unnecessary. Again, we can do either periodic updating of $\Delta_{j}\left(\hat{M}_{j}, n\right)$, or 
dynamically determine when to update $\Delta_{j}\left(\hat{M}_{j}, n\right)$ based on the change of $\hat{\boldsymbol{\theta}}_{j}(n)$. For the latter, we note that if the value of $\hat{\boldsymbol{\theta}}_{j}(n)$ unchanged, the corresponding virtual systems will be the same. Thus, we can measure the change of $\hat{\boldsymbol{\theta}}_{j}(n)$ over time, and only update $\Delta_{j}\left(\hat{M}_{j}, n\right)$ when the change has deemed significant to trigger an update of $\Delta_{j}\left(\hat{M}_{j}, n\right)$.

c) "Heat-up" period for threshold comparison: Due to few samples in the initial collision counting, the value $K_{j}\left(n ; \hat{M}_{j}, \boldsymbol{\theta}\right) / \log \left(n-n_{o}\right)$ since the last update of $\hat{M}_{j}$ may have a high variation, causing frequent update of $\hat{M}_{j}$. To avoid this, we set a "heat-up" window period $W$ to collect initial collision counts, and only do threshold comparison when $n-n_{o}>W$. Within the window $W, \hat{M}_{j}$ will remain unchanged.

4) A Network with Changing Population: In all existing studies so far, the total number of the secondary users $M$ is assumed fixed, either known or unknown. Since in practical applications there are scenarios that a secondary user can either join or leave the network, this assumption may not always hold. Therefore, there is a need for learning and access policies which are able to track the changes of $M$ as well.

Our proposed $\pi_{\mathrm{DT}}$ policy can automatically track such population change in the network, as it allows both upward and downward change of $\hat{M}_{j}$, by testing the current collision counts against the dynamic threshold. In the simulation section, we will provide an example to demonstrate such tracking behavior.

\section{Simulation Results}

In this section, we present the simulation results obtained by using $\pi_{\mathrm{DT}}$ policy developed in this paper. We assume a cognitive radio network with $N=9$ channels and $M=4$ secondary users. The channel availability $X_{i}(n)$ follows i.i.d. Bernoulli random process, for $i=1, \cdots, N$.

To demonstrate the difference of overestimation and underestimation of $M$, we plot the two cases in Fig. 1. We set the mean channel availability vector $\boldsymbol{\theta}=[0.1,0.2, \cdots, 0.9]^{T}$. We employ $\rho^{\mathrm{RAND}}$ policy, assuming a fixed estimate $\hat{M}_{j}$ as $M$, and plot the normalized regret over time for $\hat{M}_{1}=\cdots=\hat{M}_{4}>M$ and $\hat{M}_{1}=\cdots=\hat{M}_{4}<M$. As can be clearly seen, the difference in terms of regret between underestimation and overestimation is substantial when the estimate different from the true value only by 1 , i.e., $\hat{M}_{j}=M-1$ and $\hat{M}_{j}=M+1$.

In Fig. 2, we compare the obtained total regret, normalized against $\log n$, under $\pi_{\mathrm{DT}}$ with unknown $M$ and $\rho^{\text {RAND }}$ with known $M$. We also plot the regret using $\rho^{\text {RAND }}$ with a fixed wrong knowledge $\hat{M}=M-1$ or $\hat{M}=M+1$. We set the mean channel availability vector $\boldsymbol{\theta}=[0.1,0.2, \cdots, 0.9]^{T}$. As can been seen, the regret under $\rho^{\mathrm{RAND}}$ policy has logarithmic growth, as shown in [5]. For unknown $M$, under $\pi_{\mathrm{DT}}$ policy with dynamic thresholding, we see that the regret grows superlogarithmic due to the impact of $\hat{M}$ estimation error over time. Comparing to the case with fixed $\hat{M}=M-1$ which has linear growth rate, we see a large improvement in regret under the proposed $\pi_{\text {Dт }}$ policy with dynamic thresholding for $M$ estimation. The performance under the $\hat{M}=M+1$ is
Algorithm 1 : Dynamic thresholding policy $\pi_{\mathrm{DT}}$ for each user $j$, under $N$ channels and $M$ secondary users.

1) Input: $n$ : Current time slot

$T^{\prime}$ Horizon length of the virtual systems

$n_{o}$ Time slot of the latest $\hat{M}_{j}$ update

$W$ : Soft window size

$\hat{M}_{j}$ : Current estimate of $M$ by user $j$

$\hat{\theta}_{i}^{j}\left(T_{i}^{j}(n)\right)$ : Sample mean availability of channel $i$ for user $j$ up to time slot $n$

$I_{j}(i, n)$ : g-statistic of channel $i$ at time slot $n$ for user $j$ $\sigma\left(k, \mathbf{I}_{j}(n)\right)$ : Index of the $k^{t h}$ highest entry in $\mathbf{I}_{j}(n)$ $\zeta_{j}(i, n)$ :Collision indicator at time slot $\mathrm{n}$ on channel $i$

2) Init: Sense each channel once

$n \leftarrow N+1, n_{o} \leftarrow N+1, \hat{M}_{j} \leftarrow 1, r_{\text {rank }} \leftarrow 1, i_{\text {chan }} \leftarrow N$, $\zeta_{j}(i, n) \leftarrow 0$ for all channels $i=1, \cdots, N$.

3) StartLoop $n \leftarrow n+1$

4) if $\zeta_{j}\left(i_{\text {chan }}, n-1\right)=1$ then

Draw a new $r_{\text {rank }} \sim \operatorname{Unif}\left(\hat{M}_{j}\right)$ endif

Sense the channel and transmit if it is idle.

Update $i_{\text {chan }} \leftarrow \sigma\left(r_{\text {rank }}, \mathbf{I}_{j}(n)\right)$.

if $\zeta_{j}\left(i_{\text {chan }}, n-1\right)=0$ and $r_{\text {rank }}>\hat{M}_{j}$ then

Draw a new $r_{\text {rank }} \sim \operatorname{Unif}\left(\hat{M}_{j}\right)$ endif

Sense the channel and transmit if it is idle.

Update $i_{\text {chan }} \leftarrow \sigma\left(r_{\text {rank }}, \mathbf{I}_{j}(n)\right)$

5) If collision, $\zeta_{j}\left(i_{\text {chan }}, n\right) \leftarrow 1$, otherwise, 0 .

6) if $n-n_{o}>W$ then

a) Activate virtual systems $\mathcal{S}_{j u}(n)$, for $u \in\left\{\hat{M}_{j}-\right.$ $\left.1, \hat{M}_{j}\right\}$ : Set $\boldsymbol{\theta}_{j}^{\prime} \leftarrow \hat{\boldsymbol{\theta}}_{j}(n), N$ channels, run $\rho^{\mathrm{RAND}}$ policy for $T^{\prime}$ slots.

b) Update $\Delta_{j}(u, n)$, for $u \in\left\{\hat{M}_{j}-1, \hat{M}_{j}\right\}$ :

$\bar{K}\left(n^{\prime}, u, \boldsymbol{\theta}_{j}^{\prime}\right)=\frac{\sum_{j^{\prime}=1}^{u} K_{j}^{\prime}\left(n^{\prime}, u, \boldsymbol{\theta}_{j}^{\prime}\right)}{u}$

$\Delta_{j}(u, n)=\frac{\bar{K}\left(T^{\prime}, u, \boldsymbol{\theta}_{j}^{\prime}\right)}{\log T^{\prime}}$

c) Obtain total collision counts from slot $n_{o}$ up to $n$ : $K_{j}\left(n ; \hat{M}_{j}, \boldsymbol{\theta}\right)$

$\sum_{t=n_{o}+1}^{n} \sum_{k=1}^{\hat{M}_{j}} \zeta_{j}\left(\sigma\left(k, \mathbf{I}_{j}(t)\right), t\right)$.

if $\frac{K_{j}\left(n ; \hat{M}_{j}, \boldsymbol{\theta}\right)}{\log \left(n-n_{o}\right)}>\Delta_{j}\left(\hat{M}_{j}, n\right)$ then

$\hat{M}_{j} \leftarrow \hat{M}_{j}+1, n_{o} \leftarrow n$

endif

if $\frac{K_{j}\left(n ; \hat{M}_{j}, \boldsymbol{\theta}\right)}{\log \left(n-n_{o}\right)}<\Delta_{j}\left(\hat{M}_{j}-1, n\right)$ then

$\hat{M}_{j} \leftarrow \hat{M}_{j}-1, n_{o} \leftarrow n$

endif

endif

better due to a fixed overestimation. However, since $M$ is unknown, this fixed estimate cannot be ensured in reality, and the performance serves as a lower bound to our $\pi_{\mathrm{DT}}$ policy.

To see what the growth rate of regret under $\pi_{\mathrm{DT}}$, in Fig. 3, we plot the total regret normalized as $\frac{R(n, \boldsymbol{\theta}, M)}{\sqrt{n} \log n}$, which is shown approximately constant over the time horizon. This indicates that the growth rate of regret is approximately $O(\sqrt{n} \log n)$.

To show how the developed algorithm can track the change of secondary user population, we simulate a dynamic environ- 
ment in which secondary users can join or leave the network. We assume $\boldsymbol{\theta}=[0.11,0.12, \cdots, 0.19]^{T}$ and the number of secondary users $M$ varies over time from $6 \rightarrow 2 \rightarrow 5$. Fig. 4 shows the $\hat{M}_{j}$ of two secondary users who stayed in the network during the entire time. As we see, our proposed algorithm is able to track the change of total number of the secondary users in the network.

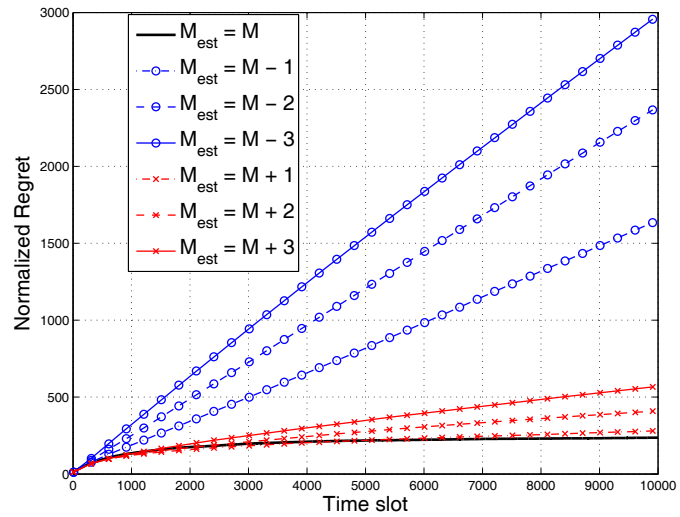

Fig. 1. Normalized regret $\frac{R(n, \boldsymbol{\theta}, M)}{\log n}$ for overestimation and underestimation of $M$.

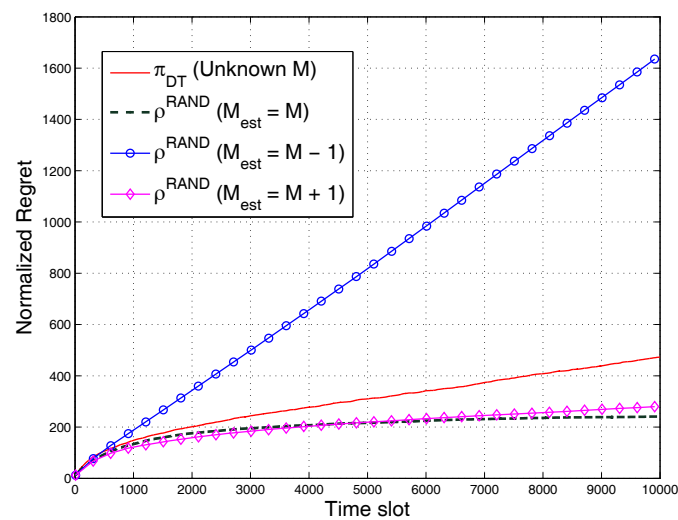

Fig. 2. Normalized regrets $\frac{R(n, \boldsymbol{\theta}, M)}{\log n}$ under $\rho^{\mathrm{RAND}}$ and $\pi_{\mathrm{DT}}$. $(\boldsymbol{\theta}=$ $[0.1,0.2, \ldots, 0.9], \mathrm{M}=4, \mathrm{~N}=9$ ).

\section{CONCLUSION}

In this paper, we developed a truly distributed dynamic spectrum access mechanism under both unknown number of secondary users $M$ and unknown mean channel availability $\boldsymbol{\theta}$. By designing thresholding mechanism for online estimation of $M$ over time, we extend $\rho^{\mathrm{RAND}}$ policy [5] to the scenario with unknown user population. We show that using distributed access policies with fixed wrong knowledge of $M$ will result in linear growth of regret over time, with underestimation incurring more significant loss than overestimation does, reflected in the rate of growth in regret. The proposed thresholding method dynamically adjusts the threshold for $M$ updates, using virtual systems built upon the current estimates of mean channel availabilities. Our algorithm allows both overestimation and underestimation in estimating $M$ over time, and thus is capable of tracking the change of $M$, i.e., population change.

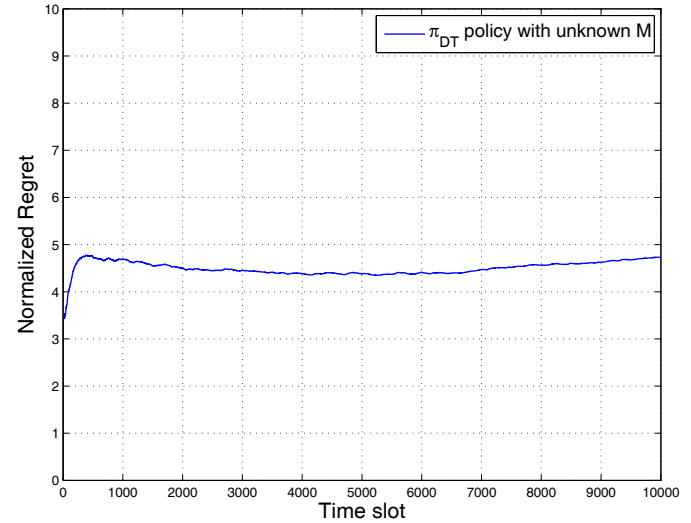

Fig. 3. Normalized regrets $\frac{R(n, \boldsymbol{\theta}, M)}{\sqrt{n} \log n}$ under $\pi_{\mathrm{DT}}(\boldsymbol{\theta}=[0.1,0.2, \ldots, 0.9], \mathrm{M}$ $=4, \mathrm{~N}=9$ ).
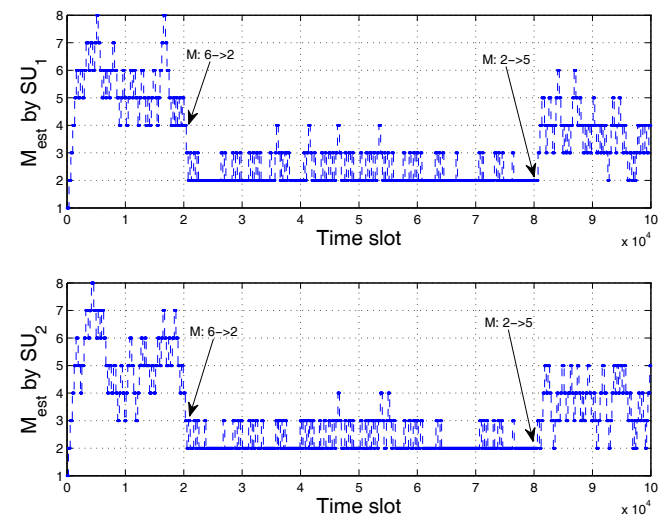

Fig. 4. Trajectory of $\hat{M}_{j}$ for secondary users in the network in a dynamic network environment $\left(\boldsymbol{\theta}=[0.11,0.12, \cdots, 0.19]^{T}, N=9\right)$

\section{REFERENCES}

[1] T. L. Lai and H. Robbins, "Asymptotically efficient adaptive allocation rules," Advances in Applied Mathematics, vol. 6, pp. 4-22, 1985.

[2] V. Anantharam, P. Varaiya, and J. Walrand, "Asymptotically efficient allocation rules for the multiarmed bandit problem with multiple playspart i: I.i.d. rewards," IEEE Trans. Automatic Control, vol. 32, no. 11, pp. 968-976, Nov. 1987.

[3] _ "Asymptotically efficient allocation rules for the multiarmed bandit problem with multiple plays- part ii: Markovian rewards," IEEE Trans. Automatic Control, vol. 32, pp. 977-982, Nov. 1987.

[4] K. Liu and Q. Zhao, "Distributed learning in multi-armed bandit with multiple player," IEEE Trans. Signal Processing, vol. 58, no. 11, pp. 5665-5681, Nov. 2010.

[5] A. Anandkumar, N. Michael, K. Tang, and A. Swami, "Distributed algorithms for learning and cognitive medium access with logarithmic regret," IEEE J. Select. Areas Commun., vol. 29, no. 4, Apr. 2011.

[6] Y. Gai, B. Krishnamachari, and M. Hsieh, "Decentralized online learning algorithms for opportunistic spectrum access," in Proc. IEEE Global Telecommunications Conference (GLOBECOM), Dec. 2011.

[7] P. Auer, N. Cesa-Bianchi, and P. Fisher, "Finite-time analysis of the multiarmed bandit problem," Machine learning, vol. 47, no. 2, pp. 235256, 2002.

[8] R. Agrawal, "Sample mean based index policies with o (logn) regret for the multi-armed bandit problem," Advances for in Applied probability, vol. 27, no. 4, pp. 1054-1078, 1995. 